\title{
Origin of spin-polarized photocurrents in the topological surface states of $\mathrm{Bi}_{2} \mathrm{Se}_{3}$
}

\author{
A. S. Ketterl, ${ }^{1}$ S. Otto, ${ }^{2}$ M. Bastian, ${ }^{1}$ B. Andres,${ }^{1}$ C. Gahl, ${ }^{1}$ J. Minár, ${ }^{3}$ H. Ebert, ${ }^{4}$ J. Braun, ${ }^{4}$ O. E. Tereshchenko, ${ }^{5,6}$ \\ K. A. Kokh, ${ }^{7,8}$ Th. Fauster, ${ }^{2}$ and M. Weinelt ${ }^{1, *}$ \\ ${ }^{1}$ Freie Universität Berlin, Fachbereich Physik, Arnimallee 14, 14195 Berlin, Germany \\ ${ }^{2}$ Friedrich-Alexander-Universität Erlangen-Nürnberg, Staudtstraße 7, 91058 Erlangen, Germany \\ ${ }^{3}$ New Technologies Research Center, West Bohemian University, Univerzitni 8, Plzen, Czech Republic \\ ${ }^{4}$ Ludwig-Maximilians-Universität München, Butenandtstraße 5-13, 81377 München, Germany \\ ${ }^{5}$ Novosibirsk State University, 630090 Novosibirsk, Russian Federation \\ ${ }^{6}$ Rzhanov Institute of Semiconductor Physics and Novosibirsk State University, 630090 Novosibirsk, Russia \\ ${ }^{7}$ Tomsk State University, 634050 Tomsk, Russia \\ ${ }^{8}$ Sobolev Institute of Geology and Mineralogy SB RAS and Novosibirsk State University, 630090 Novosibirsk, Russia
}

(Received 7 February 2018; revised manuscript received 11 September 2018; published 8 October 2018)

\begin{abstract}
For the generation of spin-polarized photocurrents in topological insulators, a coupling between photon angular momentum and electron spin is often assumed. Such a coupling seems to be supported by dichroism reported in $E\left(k_{y}\right)$-intensity maps in photoemission. We show in three dimensional two-photon photoemission and one-step photoemission calculations that the circular dichroism is in fact threefold in $E\left(k_{x}, k_{y}\right)$ maps although it may appear antisymmetric in $E\left(k_{y}\right)$. The threefold symmetry is inconsistent with the previously assumed coupling between photon momentum and electron's chiral spin via the orbital momentum. Instead it reflects the surface point group. The only antisymmetric patterns appear in the energy range in which surface and bulk states hybridize. In general, a threefold-symmetric dichroic signal does not support unidirectional photocurrents. Nevertheless, the residual asymmetry of up to $3.5 \%$ in our photoemission spectra is compatible with previously observed helicity-dependent photocurrents.
\end{abstract}

DOI: 10.1103/PhysRevB.98.155406

\section{INTRODUCTION}

Topological insulators (TIs) are one representative of topological matter with intrinsic properties promising for spintronic applications [1-8]. The topological surface states (TSSs) of three-dimensional TIs exhibit the linear dispersion of a Dirac cone. Spin and momentum are locked, i.e., the spins are oriented perpendicular to the electron's momentum and form a helical spin texture [9]. One direct consequence of spin-momentum locking is that currents flowing in the TSS are spin polarized. $\mathrm{Bi}_{2} \mathrm{Se}_{3}$ is the primary TI, for which helicity-dependent photocurrents were observed upon excitation with near-infrared (NIR) frequencies [10,11]. Picoampère (pA) photocurrents were assigned to originate from the TSSs [12] suggesting that they are indeed spin polarized. In complete contrast, experimental studies using $\mathrm{THz}$ excitation or recording $\mathrm{THz}$ emission attribute photocurrents to anisotropic carrier scattering [13] or charge shifts [14] questioning in either case their spin-polarized character. Recent photoemission studies show again evidence on transient photocurrents driven by direct optical excitation with midinfrared pulses in the TSS of $\mathrm{Sb}_{2} \mathrm{Te}_{3}[15,16]$. Transport measurements in electrically gated $\left(\mathrm{Bi}_{1-x} \mathrm{Sb}_{x}\right)_{2} \mathrm{Te}_{3}$ thin films identify the circular photogalvanic effect as the dominant source for helicitydependent photocurrents $[17,18]$. The observed pA currents are attributed to optical transitions between the topological

*weinelt@physik.fu-berlin.de surface state and bulk bands [17]. This conclusion is in line with a generic theoretical model predicting that helicitydependent photogalvanic currents are strongly suppressed when considering only the Dirac cone of TIs [19].

The narrow band gap and intrinsic doping of most threedimensional TIs and the resulting bulk conductivity make the investigation and application of surface currents difficult. This is also true for the first TSS of $\mathrm{Bi}_{2} \mathrm{Se}_{3}$, the spectral function of which is depicted in Fig. 1(a). Since $\mathrm{Bi}_{2} \mathrm{Se}_{3}$ is intrinsically $n$ doped, the Fermi level $E_{F}$ lies close above the conduction band (CB) bottom and the first TSS is fully occupied. However, Niesner et al. have shown that $\mathrm{Bi}_{2} \mathrm{Se}_{3}$ exhibits an additional unoccupied TSS between the second and third CB, $1.7 \mathrm{eV}$ above the occupied TSS [20]. Hence, photoexciting carriers from the first to the second TSS may be the underlying mechanism driving spin-polarized photocurrents with NIR pulses insensitive to details of the bulk doping and position of $E_{F}$ [17]. Note that these optically excited currents are not addressed by $\mathrm{THz}$ excitation [13].

Lateral photocurrents correspond to asymmetric carrier populations in momentum space, which can be directly mapped by means of angle-resolved two-photon photoemission (2PPE) using femtosecond laser pulses [21]. In contrast to photocurrent signals and $\mathrm{THz}$ emission, 2PPE is state selective and allows us to directly reveal the photocurrentcarrying states $[15,16]$. Our pump-probe approach is illustrated in Fig. 1. The circularly polarized NIR pump pulse excites carriers from the first to the second TSS of $\mathrm{Bi}_{2} \mathrm{Se}_{3}$, while the $s$-polarized ultraviolet (UV) probe pulse maps the 

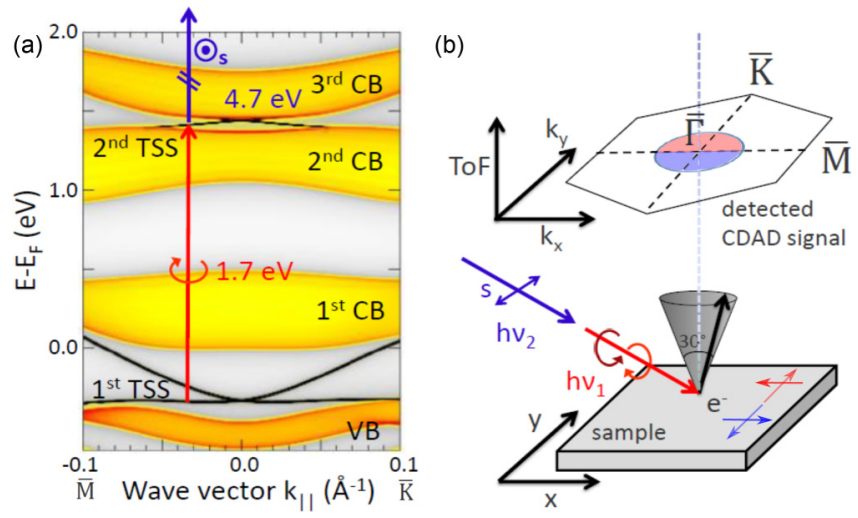

FIG. 1. (a) Spectral function of $\mathrm{Bi}_{2} \mathrm{Se}_{3}$ : The red arrow indicates interband transitions between first and second TSSs for resonant excitation $(h v=1.7 \mathrm{eV})$ with left or right circularly polarized light. An $s$-polarized laser pulse ( $h v=4.7 \mathrm{eV}$, blue arrow) was used to map the electron distribution. (b) Experimental geometry: Pump and probe beams come in along $\overline{\Gamma M}$ at an angle of $45^{\circ}$ to record CDAD patterns in a cone around the surface normal with $\pm 15^{\circ}$ opening angle.

$\left(k_{x}, k_{y}\right)$-momentum distribution of photoelectrons onto a position sensitive detector. The polarization of the probe pulse is chosen to achieve a uniform photoemission from the excited states. Patterns of the circular dichroism in the angular distribution (CDAD) are obtained by subtracting individual photoelectron intensity maps excited with left and right circularly polarized pump pulses normalized to the combined photoelectron intensity,

$$
I_{\mathrm{CDAD}}=[I(\circlearrowleft)-I(\circlearrowright)] /[I(\circlearrowleft)+I(\circlearrowright)] .
$$

If circularly polarized light incident in the $x$ direction couples to the spin texture of the TSS during excitation, the CDAD signal at corresponding (kinetic) energy would be asymmetric with respect to $k_{y}$ momentum, reflecting a spin-polarized current in the $y$ direction.

In the present study, we compare energy-selective $\left(k_{x}, k_{y}\right)$ momentum maps recorded by $2 \mathrm{PPE}$ with one-step photoemission calculations. Using circularly polarized pulses with 1.7 and $1.9 \mathrm{eV}$ photon energy, electrons were excited resonantly and off resonantly from the occupied to the unoccupied TSS of $\mathrm{Bi}_{2} \mathrm{Se}_{3}[20,22,23]$. We find that the dichroic signal originates from the initial states, i.e., the pump step in 2PPE. Therefore our results are directly comparable to the measurements of lateral photocurrents $[10,11]$. The observed CDAD patterns of the occupied TSS reflect the crystal symmetry. Evaluating the asymmetry with respect to $k_{y}$ momentum we observe residual asymmetries of $\leqslant 3.5 \%$ in our photoemission experiment. These asymmetries are compatible with photocurrents in the picoampère range $[10,17]$. However, contrary to previously reported results [10] asymmetries in $k_{x}$ and $k_{y}$ directions, i.e., photocurrents running parallel or perpendicular to the photon angular momentum, have the same order of magnitude. In line with the threefold symmetry of the CDAD patterns, calculated photoemission maps show negligible asymmetry $(\leqslant 0.09 \%)$. Only in the energy range where surface and bulk states, which are neither topologically protected nor a priori spin polarized, hybridize do we observe patterns indicating lateral photocurrents in the $k_{y}$ direction.

\section{EXPERIMENT}

To generate ultrashort infrared pump pulses, an optical parametric amplifier driven by a regenerative Ti:Sa amplifier was used. The circular polarization of the pump pulses was achieved with a quarter-wave plate in combination with a Soleil-Babinet compensator to precompensate polarization changes induced by following mirrors and the window of the vacuum chamber. The probe pulse with a photon energy of $4.7 \mathrm{eV}$ was obtained by frequency tripling of the Ti:Sa fundamental. The UV photon energy is not sufficient to overcome the $\mathrm{Bi}_{2} \mathrm{Se}_{3}$ work function of $5.61 \mathrm{eV}$ [23], but enough to fully access the unoccupied TSS. Pump and probe pulses had a cross correlation of $80 \mathrm{fs}$. Photoelectrons were analyzed by an angle-resolving time-of-flight (ToF) spectrometer (Themis, SPECS GmbH), which allowed for simultaneous recording of their kinetic energy and parallel momentum $\left(k_{x}, k_{y}\right)$ with a polar acceptance angle of $\pm 15^{\circ}$.

$\mathrm{Bi}_{2} \mathrm{Se}_{3}$ crystals were grown in sealed quartz ampules coated with a carbon layer from elementary Bi and Se with 99.999\% purity by a vertical variant of the modified Bridgman method. The resulting ingots consisted of one or several single-crystalline blocks and were naturally $n$ doped with carrier concentrations in the range of $10^{18}-10^{19} \mathrm{~cm}^{-3}$. The samples were cleaved at $2 \times 10^{-9} \mathrm{mbar}$ and transferred to our photoemission chamber with a base pressure of $6 \times$ $10^{-11}$ mbar within less than $2 \mathrm{~min}$. There, the samples were kept at $100 \mathrm{~K}$ throughout the entire measurement. Sample orientation and surface order were controlled by low-energy electron diffraction.

\section{THEORY}

Self-consistent electronic structure calculations were performed within the $a b$ initio framework of spin-density functional theory using Vosko, Wilk, and Nusair parametrization for the exchange and correlation potential [24]. The electronic structure was calculated in a fully relativistic way by solving the corresponding Dirac equation using the relativistic multiple-scattering formalism in the tight-binding KorringaKohn-Rostocker (KKR) mode [25,26]. The resulting halfspace electronic structure represented by single-site scattering matrices for the different layers and the corresponding wave functions for initial- and final-state energies serve as input quantities for the corresponding photocurrent calculations. They are based on the fully relativistic one-step model in its spin-density matrix formulation [27]. This approach allows us to properly describe the complete spin-polarization vector. We include a Rundgren-Malmström-type surface potential [28] into the formalism as an additional layer to obtain the correct description of the energetics and dispersion of all surfacerelated features [cf. Fig. 1(a)]. Furthermore, the relative intensities of surface states and resonances are quantitatively accounted for by calculating the corresponding matrix elements in the surface region [29]. The energy-dependent retarded KKR Green's function which represents the initial state has been calculated for complex energies $E$, with a constant 

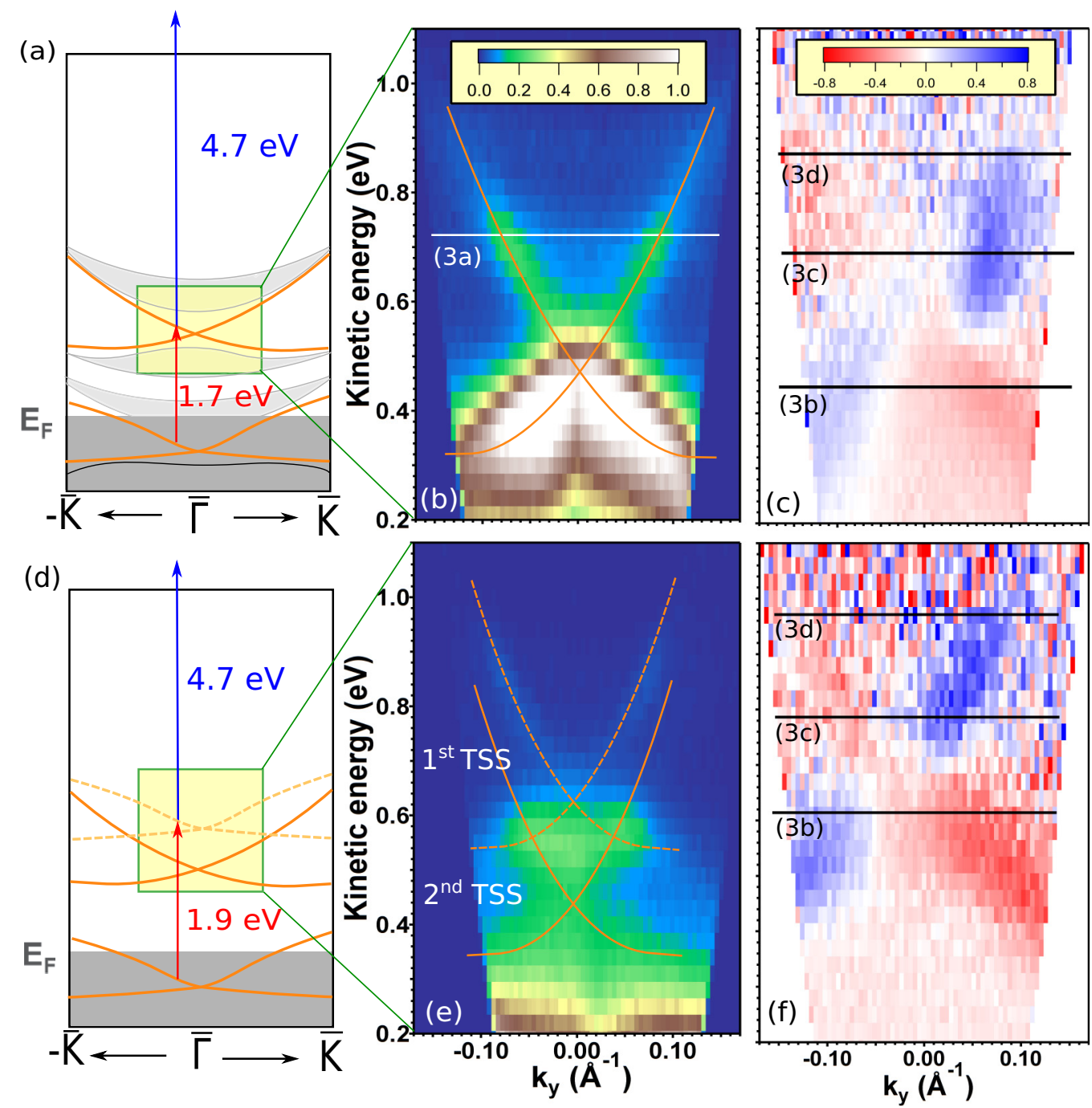

FIG. 2. (a),(d) Sketch of the 2PPE process for resonant (upper row) and off-resonant (lower row) excitation from the first to the second TSS. Dashed lines indicate the virtual intermediate state. Yellow highlighted rectangles in (a) and (d) mark the energy vs $k_{y}$ range of the displayed 2PPE maps. (b),(e) 2PPE intensity along $\pm \overline{\Gamma K}$ for excitation with unpolarized NIR light and probing with $s$-polarized UV pulses. Orange lines mark the expected position of the TSSs. (c),(f) CDAD resulting from excitation with circularly polarized pulses at 1.7 and $1.9 \mathrm{eV}$ photon energy, respectively. The left and right branches of the first TSS exhibit an asymmetry of up to $60 \%$.

imaginary part $V_{i}(E)=0.004 \mathrm{eV}$, to account for damping effects due to inelastic scattering events. This way the finite lifetime of the initial state has been considered. Also in the final-state calculation many-body effects have been included phenomenologically by use of a weakly energy-dependent, complex inner potential [30,31].

\section{RESULTS AND DISCUSSION}

Figure 2 comprise 2PPE schemes and intensity maps for resonant (upper row) and off-resonant (lower row) excitation from the first to the second TSS. All 2PPE maps show photoemission perpendicular to the plane of incidence of the light, i.e., along $\bar{\Gamma}-\overline{\mathrm{K}}\left( \pm k_{y}\right.$-direction). The $2 \mathrm{PPE}$ process describing resonant excitation is sketched in Fig. 2(a). The rectangle highlighted in yellow marks the energy vs $k_{y}$ section depicted in Figs. 2(b) and 2(c) (upper row). Upon excitation with unpolarized pulses of $1.7 \mathrm{eV}$ photon energy and probing with $s$-polarized pulses of $4.7 \mathrm{eV}$ photon energy, the $2 \mathrm{PPE}$ map shows the signature of a single Dirac cone [highlighted by the orange line in Fig. 2(b)]. Additionally to the TSS, a strong 2PPE signal is obtained for transitions from the VB to the second $\mathrm{CB}$ overlaying the Dirac point at $0.4 \mathrm{eV}$ kinetic energy, and a much weaker signal around $0.9 \mathrm{eV}$ corresponding to excitations from the occupied part of the first $\mathrm{CB}$ into the third CB. Detuning the unpolarized NIR pump pulse to $1.9 \mathrm{eV}$ for off-resonant excitation reveals signatures of both TSSs [Figs. 2(d) and 2(e), lower row]. The 2PPE signal of the occupied first TSS (initial state) is displaced by $0.2 \mathrm{eV}$ to higher kinetic energy. The position of the unoccupied second TSS (intermediate state) remains unchanged compared to resonant excitation since it only depends on the probe pulse photon energy. As expected, off-resonant excitation results in an overall decrease of the 2PPE signal of both TSSs. These results prove that resonant excitation between the two topological surface states is possible at $1.7 \mathrm{eV}$ photon energy. Figures 2(b) and 2(d) show that excitation with unpolarized light leads to an equal intensity of the left and right $k_{y}$ branch 
(a) unpolarized excitation
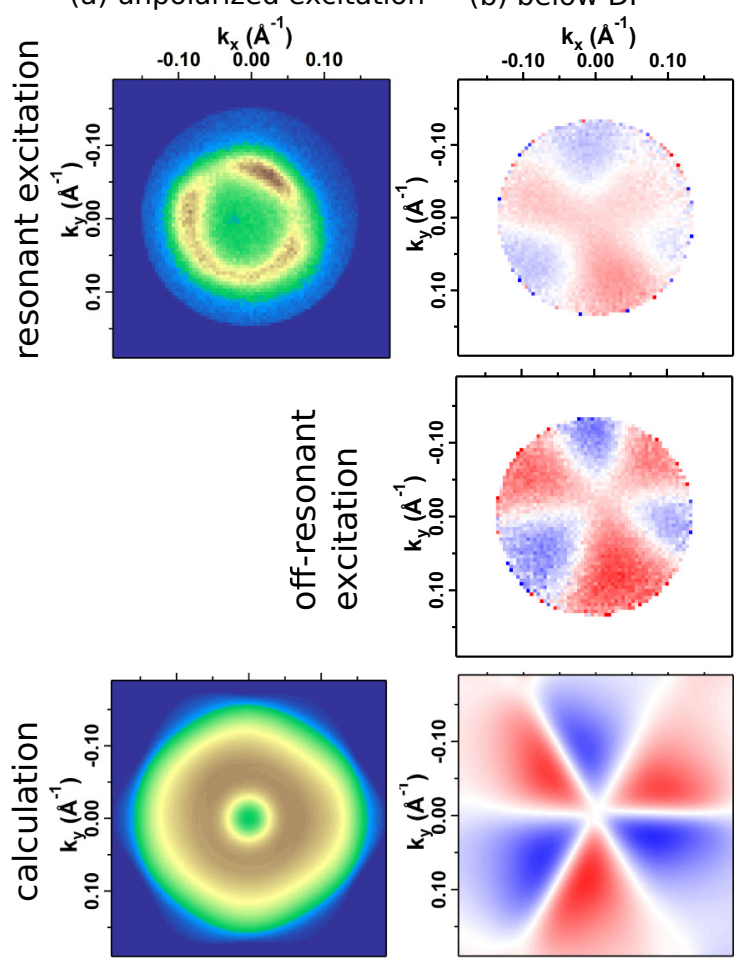

(c) above DP

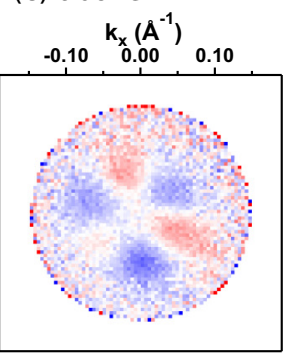

(d) at CB bottom
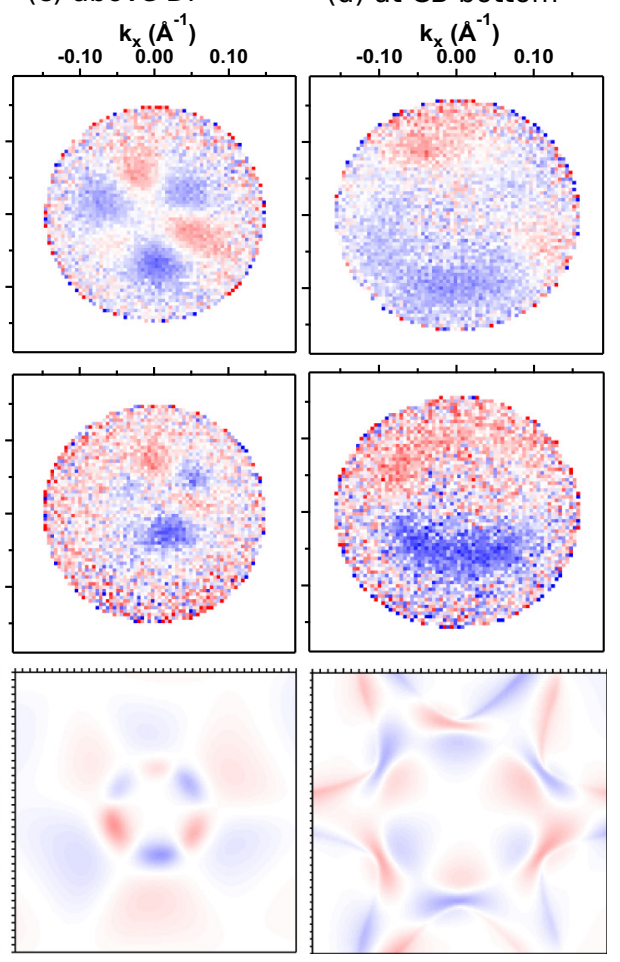

FIG. 3. 2PPE and CDAD maps of the first TSS for sections in the $\left(k_{x}, k_{y}\right)$ parallel momentum plane at selected kinetic energies $E_{\text {kin }}$ indicated by the horizontal lines in Fig. 2. The experimental maps were averaged over an energy range of $E_{\text {kin }} \pm 15 \mathrm{meV}$. Upper and middle row show maps for resonant $(h v=1.7 \mathrm{eV})$ and off-resonant $(h v=1.9 \mathrm{eV})$ excitation, respectively. $s$-polarized probe pulses of $4.7 \mathrm{eV}$ photon energy were used. The lower row shows patterns obtained from one-step photoemission calculations (see text). Column (a): 2PPE map for unpolarized excitation. Columns (b) and (c): CDAD patterns extracted below and above the Dirac point (DP) of the first TSS. Column (d): CDAD pattern observed at the bottom of the first $\mathrm{CB}$.

of the Dirac cone. Even more important, the probe step with $s$-polarized UV light does not affect the angular distribution. Furthermore, previous studies have shown that the final states for our excitation energy have $s$-like character [32-35], so their influence on the circular dichroism should be minimal.

The dichroism observed by 2PPE upon pumping with circularly polarized light is unaffected by the probe step and reveals the transient (de)population of the TSSs. CDAD maps, Eq. (1), for resonant and off-resonant excitation are depicted in Figs. 2(c) and 2(f), respectively. The opposite branches of the first TSS, which have opposite spin polarization, exhibit a strong opposite dichroic contrast of 20-60\%. This observation is similar to the results of Kuroda et al. [15,16] and would support the hypothesis of the light coupling predominantly to electrons whose spin corresponds to the photon angular momentum [10]. For off-resonant excitation the CDAD of the first occupied TSS remains, but we do not observe a dichroic contrast for the second unoccupied TSS. Resonant excitation from the VB and/or ultrafast repopulation of the unoccupied TSS by electrons excited to the second and third CBs may diminish the CDAD. However, since photoexcited holes in the occupied TSS have about 10-100 times longer lifetime [11] $\tau$ compared to the photoexcited electrons in the second TSS [23], the photocurrents will be mainly carried by holes. In conclusion, the CDAD patterns recorded along $\pm k_{y}$, i.e., a single azimuth, would suggest that the previously observed photocurrents $[10,11]$ in $\mathrm{Bi}_{2} \mathrm{Se}_{3}$ result from excit- ing the occupied TSS and are insensitive to the exact NIR photon energy. These currents could be indeed strongly spin polarized.

However, to identify the true origin of the circular dichroism it is essential to study the complete CDAD pattern [20]. Figure 3 shows cuts in the $\left(k_{x}, k_{y}\right)$ parallel momentum plane for selected kinetic energies indicated by the horizontal lines in Fig. 2. Upper and middle rows comprise patterns of the first TSS recorded for resonant and off-resonant excitation, respectively. The lower row shows corresponding one-step photoemission calculations. The two panels in column (a) of Fig. 3 depict a cut through the upper branch of the TSS for excitation with unpolarized light. The 2PPE pattern is fairly isotropic, but shows a small threefold modulation of the intensity, which is a signature of a weak warping of the Dirac cone. This small deviation from the circular shape is also perceivable in the calculations (bottom row) for direct photoemission with $4.7 \mathrm{eV}$ photon energy from the second Dirac cone. These calculations assume an isotropic population of the second TSS (intermediate state) and therefore confirm that the $s$-polarized probe pulse does not influence the CDAD patterns.

The full CDAD maps in Fig. 3 reveal clear threefold symmetric patterns both at energies below and above the Dirac point of the first TSS: Below the Dirac point [Fig. 3, column (b)], a strong threefold pattern can be seen that extends uniformly over the entire measured $k_{\|}$range. Above the Dirac 
point [Fig. 3, column (c)], a threefold pattern of reversed orientation shows up at the constant energy surface of the TSS. Such threefold symmetric patterns, provided they are perfect, would not lead to a photocurrent, since the dichroic contribution along any arbitrary in-plane direction $\mathbf{k}_{\|}$cancels with the sum of the by $\pm 120^{\circ}$ rotated, symmetry-equivalent components projected along $-\mathbf{k}_{\|}$and vice versa. Therefore it is not sufficient to record helicity-dependent photoemission signals along a fixed azimuth $\pm \mathbf{k}_{\|}$to prove the origin of spin currents. Instead, the threefold patterns reflect the bulk symmetry, even though the initial state is of surface character. For off-resonant excitation (middle row) the CDAD signal from the first, occupied TSS manifests very strong similarities to the case of resonant excitation (upper row). In contrast, no distinct features in the energy range that corresponds to photoelectrons from the second, unoccupied TSS were observed [cf. Figs. 2(d)-2(f)]. Antisymmetric patterns, the signature of photocurrents, can only be seen in energy ranges in which the TSS hybridizes with the bulk bands [Fig. 3, column (d)]. In all cases the CDAD results from excitation of the initial states by the pump pulse. This is corroborated by time-resolved measurements (see Supplemental Material [36]): CDAD is only observed when pump and probe pulses overlap. It vanishes at pump-probe delays exceeding the 80-fs cross correlation, when the 2PPE signal is dominated by the intermediate state [37].

As is evident from closer inspection of Fig. 3 the measured CDAD patterns are not perfectly threefold symmetric and these deviations can support currents. To relate our photoemission data to transport measurements, we estimated the photocurrent density $j\left(k_{y}\right)$ along $k_{y}$, i.e., perpendicular to the incoming laser beam, via the helicity-dependent asymmetry $A\left(k_{y}\right)$,

$$
\begin{aligned}
A\left(k_{y}\right) & =\frac{\sum_{\mathbf{k}_{\|}} I_{\mathrm{CDAD}}\left(\mathbf{k}_{\|}\right) \cos \varphi_{k_{y}}}{\sum_{\mathbf{k}_{\|}} \cos \varphi_{k_{y}}}, \quad \varphi_{k_{y}}=\measuredangle k_{y}, \mathbf{k}_{\|}, \\
j\left(k_{y}\right) & =n_{\text {ex }} \operatorname{ev} A\left(k_{y}\right) .
\end{aligned}
$$

The $\cos \varphi_{k_{y}}$ projects the momentum $\mathbf{k}_{\|}$onto the $k_{y}$ direction, which accounts for the projection of the group velocity $v$ of the carriers onto the $y$ direction. From the two measured CDAD patterns of the Dirac cone [Figs. 3(b) and 3(c)] we derive maximal asymmetries $A\left(k_{y}\right)=3.5 \%$, while those in $k_{x}$ are somewhat smaller, $A\left(k_{x}\right)=1.8 \%$. In the range where the TSS hybridizes with the bulk bands the asymmetry is more pronounced $\left[A\left(k_{y}\right)=5.6 \%\right.$ vs $\left.A\left(k_{x}\right)=0.5 \%\right]$, as is also obvious from Fig. 3(d).

In the following, we present an estimation of the photocurrent resulting from the residual asymmetry, based on upper limits for the total density of excited carriers and for the photohole lifetimes. Furthermore, we take it as read that the slope of the Dirac cone is constant $\hbar v \sim 3.2 \mathrm{eV} \AA$ (cf. Fig. 2). As an upper limit we assume that $1 \%$ of the electrons in the occupied Dirac cone $n \sim 2 \times 10^{13} \mathrm{~cm}^{-2}$ are photoexcited by the pump pulse $n_{e x}=0.01 n$. This yields total surface-photocurrent densities of $j=2-3 \times 10^{2} e \mathrm{~cm}^{-1} \mathrm{fs}^{-1}$. The typical lifetimes of photoexcited carriers in the TSS close to the Fermi level, i.e., the momentum-dependent population decay times, are in the order of 1 ps [16,22,38], leading to around $10^{5}$ excited electrons/cm per laser pulse. Finally, with a laser repetition rate of $300 \mathrm{kHz}$ and spot diameter of $200 \mu \mathrm{m}$ we estimate a photocurrent of $10^{8} \mathrm{e} / \mathrm{s}$, which corresponds to $10 \mathrm{pA}$. We note that the pump-pulse intensity and its average power $(<100 \mathrm{~mW})$ in our photoemission experiment are comparable to those used in reported photocurrent measurements $[10,11,17]$. Therefore, the asymmetry of the measured CDAD patterns is compatible with the reported picoampère photocurrents. Our time-resolved measurements show that the CDAD patterns in the excited states persists for more than 50 fs (cf. the Supplemental Material [36]), making the estimated photocurrent still on the order of a picoampère and therefore comparable to the currents observed by McIver et al. [10], especially if we assume slightly higher excitation densities.

Generally, the circular dichroism from TIs stems from the complex interplay between the involved initial, intermediate, and final states [32]. To further test the influence of the intermediate state, we compare our results to one-step photoemission calculations with the combined photon energy of $6.4 \mathrm{eV}$. This corresponds to a direct transition from initial to final states. The obtained theoretical predictions for CDAD patterns are compiled in the bottom row of Fig. 3. We find very good agreement for photoemission from the lower and upper branch of the first TSS, which supports our hypothesis that the recorded CDAD patterns reflect the coupling of the circularly polarized photons to the orbital symmetry of the occupied TSS. However, the calculations are not able to reproduce the asymmetric patterns we observe close to the Fermi level. This indicates that these patterns include the complicated physical nature of the intermediate state. This state in principle must be described by the two-time dependent lesser Green's function and not by the much simpler retarded Green's function which is typically used in a one-step photoemission calculation [39]. Note that the lifetime of excited electrons in the intermediate state is only $\tau \sim 70 \mathrm{fs}$ (see Ref. [23] and the Supplemental Material [36]) and photocurrents are dominated by holes generated in the initial state. The calculated dichroism patterns show very small asymmetries of $0.09 \%$ and $0.02 \%$ projected onto the $k_{y}$ and $k_{x}$ direction, respectively. We attribute these to residuals from numerical discretization unavoidable in quantitative calculations rather than to an intrinsic property of the CDAD. This suggests that the experimentally determined asymmetries equally stem from small experimental errors. This can also explain the observed asymmetries along the plane of incidence of the light, which are in principle symmetry forbidden. As our experiments are performed under UHV conditions and we do not observe surface band bending [40], we cannot exclude Rashba surface states as the source of photocurrents observed under ambient conditions. However, we want to emphasize that our measurements exclude the TSSs as the primary source.

\section{CONCLUSION}

In conclusion, the circular dichroism we observe for the TSSs of $\mathrm{Bi}_{2} \mathrm{Se}_{3}$ stems from coupling of the circularly polarized light of 1.7 and $1.9 \mathrm{eV}$ photon energy to the initial states. Neither the intermediate TSS nor the final states or the $s$-polarized probe photons influence these patterns. The CDAD patterns therefore reflect the excitation patterns of 
$\mathrm{Bi}_{2} \mathrm{Se}_{3}$ with circularly polarized light. The CDAD patterns we observe from the occupied Dirac cone show threefold rotational symmetry and are insensitive to the exact excitation energy. We measure asymmetries of below 3.5\%. From this, we can estimate surface photocurrents in the picoampère range, which are in agreement with previously reported photocurrents. This is mutually reinforcing, since both the estimation of macroscopic currents from 2PPE and the pinpointing of current origins from transport measurements are rather involved. Based on the excellent agreement between measured dichroism patterns of the occupied Dirac cone and onestep photoemission calculations, we conclude that helicitydependent photocurrents do not reflect an intrinsic property of the topological surface state of $\mathrm{Bi}_{2} \mathrm{Se}_{3}$. Furthermore, we emphasize that the threefold symmetrical excitation patterns in the energy range of the TSS can only be observed by recording the full parallel momentum range, while cuts along a single $\mathbf{k}_{\|}$direction are misleading. Indeed for a threefold symmetric pattern a $\pm k_{y}$ asymmetry can be expected. However, a current along $+k_{y}$ is canceled by the projection of the two threefold-symmetry-equivalent components onto the $-k_{y}$ direction. We only observe an antisymmetric twofold excitation pattern at the CB bottom, where the TSS hybridizes with bulk states. Since these states are not symmetry protected they carry photocurrents which are a priori not spin polarized.

\section{ACKNOWLEDGMENTS}

Support by the Deutsche Forschungsgemeinschaft (DFG) and the TRR 227 "Ultrafast Spin Dynamics" is gratefully acknowledged. A.S.K. is indebted to the International Max Planck Research School Functional Interfaces in Physics and Chemistry for financial support. Financial support by the DFG SPP1666 priority program (Grant No. EB154/32) and by Bundesministerium für Bildung und Forschung (BMBF) Project No. 05K16WMA is gratefully acknowledged. J.M. was supported by the European Regional Development Fund (ERDF), project CEDAMNF, Reg. No. CZ.02.1.01/0.0/0.0/15_003/0000358. O.T. and A.K. have been supported by the Russian Science Foundation (Project No. 17-12-01047).
[1] C. L. Kane and E. J. Mele, Phys. Rev. Lett. 95, 146802 (2005).

[2] B. A. Bernevig, T. L. Hughes, and S.-C. Zhang, Science 314, 1757 (2006).

[3] J. E. Moore and L. Balents, Phys. Rev. B 75, 121306 (2007).

[4] L. Fu, C. L. Kane, and E. J. Mele, Phys. Rev. Lett. 98, 106803 (2007).

[5] L. Fu and C. L. Kane, Phys. Rev. B 76, 045302 (2007).

[6] D. Hsieh, D. Qian, L. Wray, Y. Xia, Y. S. Hor, R. J. Cava, and M. Z. Hasan, Nature (London) 452, 970 (2008).

[7] I. Garate and M. Franz, Phys. Rev. Lett. 104, 146802 (2010).

[8] O. V. Yazyev, J. E. Moore, and S. G. Louie, Phys. Rev. Lett. 105, 266806 (2010).

[9] D. Hsieh, Y. Xia, L. Wray, D. Qian, A. Pal, J. H. Dil, J. Osterwalder, F. Meier, G. Bihlmayer, C. L. Kane, Y. S. Hor, R. J. Cava, and M. Z. Hasan, Science 323, 919 (2009).

[10] J. McIver, D. Hsieh, H. Steinberg, P. Jarillo-Herrero, and N. Gedik, Nat. Nanotechnol. 7, 96 (2012).

[11] C. Kastl, C. Karnetzky, H. Karl, and A. W. Holleitner, Nat. Commun. 6, 6617 (2015).

[12] P. Hosur, Phys. Rev. B 83, 035309 (2011).

[13] P. Olbrich, L. E. Golub, T. Herrmann, S. N. Danilov, H. Plank, V. V. Bel'kov, G. Mussler, C. Weyrich, C. M. Schneider, J. Kampmeier, D. Grützmacher, L. Plucinski, M. Eschbach, and S. D. Ganichev, Phys. Rev. Lett. 113, 096601 (2014).

[14] L. Braun, G. Mussler, A. Hruban, M. Konczykowski, M. Wolf, T. Schumann, M. Münzenberg, L. Perfetti, and T. Kampfrath, Nat. Commun. 7, 13259 (2016).

[15] K. Kuroda, J. Reimann, J. Güdde, and U. Höfer, Phys. Rev. Lett. 116, 076801 (2016).

[16] K. Kuroda, J. Reimann, K. A. Kokh, O. E. Tereshchenko, A. Kimura, J. Güdde, and U. Höfer, Phys. Rev. B 95, 081103 (2017).

[17] Y. Pan, Q.-Z. Wang, A. L. Yeats, T. Pillsbury, T. C. Flanagan, A. Richardella, H. Zhang, D. D. Awschalom, C.-X. Liu, and N. Samarth, Nat. Commun. 8, 1037 (2017).
[18] D.-X. Qu, X. Che, X. Kou, L. Pan, J. Crowhurst, M. R. Armstrong, J. Dubois, K. L. Wang, and G. F. Chapline, Phys. Rev. B 97, 045308 (2018).

[19] A. Junck, G. Refael, and F. von Oppen, Phys. Rev. B 88, 075144 (2013).

[20] D. Niesner, Th. Fauster, S. V. Eremeev, T. V. Menshchikova, Y. M. Koroteev, A. P. Protogenov, E. V. Chulkov, O. E. Tereshchenko, K. A. Kokh, O. Alekperov, A. Nadjafov, and N. Mamedov, Phys. Rev. B 86, 205403 (2012).

[21] J. Güdde, M. Rohleder, T. Meier, S. W. Koch, and U. Höfer, Science 318, 1287 (2007).

[22] J. A. Sobota, S. Yang, J. G. Analytis, Y. L. Chen, I. R. Fisher, P. S. Kirchmann, and Z.-X. Shen, Phys. Rev. Lett. 108, 117403 (2012).

[23] D. Niesner, S. Otto, Th. Fauster, E. Chulkov, S. Eremeev, O. Tereshchenko, and K. Kokh, J. Electron Spectrosc. Relat. Phenom. 195, 258 (2014).

[24] S. H. Vosko, L. Wilk, and M. Nusair, Can. J. Phys. 58, 1200 (1980).

[25] H. Ebert, D. Ködderitzsch, and J. Minár, Rep. Prog. Phys. 74, 096501 (2011).

[26] H. Ebert, M. Battocletti, D. Benea, S. Bornemann, J. Braun, S. Chadov, M. Deng, H. Freyer, T. Huhne, D. Ködderitzsch, M. Kosuth, S. Lowitzer, S. Mankovskyy, J. Minar, A. Perlov, V. Popescu, O. Sipr, and Ch. Zecha, The Munich SPR-KKR package, version 7.7, 2017, http://olymp.cup.unimuenchen.de/ak/ebert/SPRKKR.

[27] J. Braun, K. Miyamoto, A. Kimura, T. Okuda, M. Donath, H. Ebert, and J. Minár, New J. Phys. 16, 015005 (2014).

[28] G. Malmström and J. Rundgren, Comput. Phys. Commun. 19, 263 (1980).

[29] A. Nuber, J. Braun, F. Forster, J. Minár, F. Reinert, and H. Ebert, Phys. Rev. B 83, 165401 (2011).

[30] J. B. Pendry, Low Energy Electron Diffraction (Academic Press, London, 1974). 
[31] G. Hilgers, M. Potthoff, N. Müller, U. Heinzmann, L. Haunert, J. Braun, and G. Borstel, Phys. Rev. B 52, 14859 (1995).

[32] J. Sánchez-Barriga, A. Varykhalov, J. Braun, S.-Y. Xu, N. Alidoust, O. Kornilov, J. Minár, K. Hummer, G. Springholz, G. Bauer, R. Schumann, L. V. Yashina, H. Ebert, M. Z. Hasan, and O. Rader, Phys. Rev. X 4, 011046 (2014).

[33] Y. Ishida, H. Kanto, A. Kikkawa, Y. Taguchi, Y. Ito, Y. Ota, K. Okazaki, W. Malaeb, M. Mulazzi, M. Okawa, S. Watanabe, C.-T. Chen, M. Kim, C. Bell, Y. Kozuka, H. Y. Hwang, Y. Tokura, and S. Shin, Phys. Rev. Lett. 107, 077601 (2011).

[34] H. Zhang, C.-X. Liu, and S.-C. Zhang, Phys. Rev. Lett. 111, 066801 (2013).

[35] S. R. Park, J. Han, C. Kim, Y. Y. Koh, C. Kim, H. Lee, H. J. Choi, J. H. Han, K. D. Lee, N. J. Hur, M. Arita, K. Shimada,
H. Namatame, and M. Taniguchi, Phys. Rev. Lett. 108, 046805 (2012).

[36] See Supplemental Material at http://link.aps.org/supplemental/ 10.1103/PhysRevB.98.155406 for time-resolved CDAD measurements.

[37] M. Weinelt, J. Phys.: Condens. Matter 14, R1099 (2002).

[38] J. Reimann, J. Güdde, K. Kuroda, E. V. Chulkov, and U. Höfer, Phys. Rev. B 90, 081106 (2014).

[39] J. Braun, R. Rausch, M. Potthoff, and H. Ebert, Phys. Rev. B 94, 125128 (2016).

[40] M. Bahramy, P. King, A. de la Torre, J. Chang, M. Shi, L. Patthey, G. Balakrishnan, Ph. Hofmann, R. Arita, N. Nagaosa, and F. Baumberger, Nat. Commun. 3, 1159 (2012). 